\title{
Caracterización de las Ocupaciones de los Períodos Intermedio Tardío y Tardío ( 1.000-1.536 años D.C.) en el Valle de Mauro (IV Región, Chile) a partir del Registro Zooarqueológico
}

\author{
Characterization of Late Intermediate and Late Period Occupations ( 1.000-1.536 \\ Years A.D.) in Mauro Valley (IV Región, Chile) Through the Zooarchaeological Record \\ Bárbara Rivera $^{\mathrm{i}}$, Patricio López Mendoza ${ }^{\mathrm{ii}}$, Isabel Cartajena ${ }^{\mathrm{iii}}$, Boris Santander ${ }^{\mathrm{iv}}$
}

\begin{abstract}
RESUMEN
Se discuten los resultados del análisis zooarqueológico de restos provenientes del Período Intermedio Tardío ( I.000-I.400 años d.C.) y el Período Tardío (I.400-I.536 d.C.) del Valle de Mauro (IV Región, Chile). Las ocupaciones PIT evidencian explotaciones de recursos del valle a baja escala y centrado en los camélidos, aspecto coherente con lo señalado para el período en otros sectores del Norte Semiárido. Para el PT los recursos se diversifican y aumentan en número lo que va de la mano con los cambios sociopolíticos y económicos implantados por el Tawantinsuyu. Dentro del registro para ambos períodos, destaca la diversificación de tamaños en los camélidos, lo que puede relacionarse a procesos de crianza locales así como la introducción de taxa domésticos por parte del Inca, y su uso en rutas caravaneras, como recurso alimenticio y para proveer de materias primas en actividades como la confección de textiles.
\end{abstract}

Palabras Clave: Período Intermedio Tardío, Período Tardío, Zooarqueología, Norte Semiárido, Chile.

\section{ABSTRACT}

The results obtained from the zooarchaeological analysis of the Late Intermediate Period ( I.000-I.400 AD) and the Late Period (I.400-I.536 AD) of the Valle de Mauroare discussed. Late

i Tesista de Pregrado en Arqueología, Departamento de Antropología, Facultad de Ciencias Sociales, Universidad de Chile. Correo-e: briveramaro@gmail.com

ii Programa de Doctorado en Antropología, Universidad Católica del Norte, Instituto de Investigaciones Arqueológicas y Museo Gustavo Le Paige (IIAM), Calle Gustavo Le Paige No 380, San Pedro de Atacama, Chile. Correo-e: patriciolopezmend@yahoo.es

iii Universidad de Chile, Departamento de Antropología, Facultad de Ciencias Sociales, Ignacio Carrera Pinto 1045, Nuñoa, Santiago, Chile. Correo-e: icartaje@uchile.cl

iv Programa de Doctorado en Cuaternario y Prehistoria, Universitat Rovira i Virgili, España; InstitutCatalà de Paleoecologia Humana i Evolució Social, IPHES, Tarragona, España. Correo-e: boris.santander@gmail.com

Recibido: 14-01-2013 Revisado: 05-12-2013 Aceptado: 14-02-2014 
Intermediate Period occupations shows a low scale resource exploitation of the valley, centered in camelids, which is coherent with what was indicated for the same period in other sectors of the Semiarid North. In the case of the Late Period, resources are diversified and they increase in number, which is related to sociopolitical and economical changes well-established by the Tawantinsuyu. Regarding the bone record for both periods there is a diversification of camelids sizes, which is probably related to local breeding processes and domestic taxa introduction by the Inca, and their use in caravan routes, as a food resource and to provide source materials in activities such as manufacture of textiles.

Key Words: Late Intermediate Period, Late Period, Zooarchaeology, Semiarid North, Chile

\section{INTRODUCCIÓN}

El Período Intermedio Tardío (PIT en adelante) en el Norte Semiárido de Chile (NSA en adelante) se caracteriza principalmente por la presencia de la Cultura Diaguita ( I.000-I.400 años d.C.), sociedad agrícola con un patrón de asentamiento disperso centrado en terrazas fluviales y que se define por una explotación a baja escala del entorno (Troncoso 1999, 200I). Bajo este escenario, destacan la presencia del estilo cerámico Diaguita I y II, la fabricación de herramientas líticas con materias primas locales, amplio consumo de quínoa y la caza predominante de guanacos (Lama guanicoe). Es importante destacar la presencia de registro malacológico en tierras interiores, principalmente macha (Mesodesmadonacium), como resultado de contactos con la costa (Troncoso 1999, 200I). En adelante, estos contextos culturales evidenciarán múltiples cambios que se encuentran plasmados en la iconografía presente en la cerámica, la cual se complejiza (González 2004), además de una mayor cantidad de piezas decoradas (Cuarto Estilo) y mayor refinamiento en su producción. En cuanto a las evidencias líticas, destaca la presencia de adornos corporales, incluso la reutilización de los tembetás del Período Alfarero Temprano (PAT en adelante), sumado a la presencia de adornos en metal (Troncoso 2004, Latorre y López 20II). En términos de subsistencia, al consumo de quínoa se suma un aumento en el consumo de maíz (Belmar y Quiroz 2004) y además, se observa una mayor presencia de guanaco y la posible aparición de llamas (Lama glama), al mismo tiempo que aumenta el consumo de peces y restos malacológicos, tanto en número como en diversidad (Becker 2004, Troncoso 2004).

Dentro de este contexto de transformaciones sociales, económicas y tecnológicas asociadas a la llegada del Inca durante el Período Tardío (PT en adelante), destacan además nuevas formas de apropiación y uso del entorno natural, como la aparición y utilización de la llama (Lama glama) como recurso econó- mico y estratégico para articular áreas distantes. Son estos cambios los que denotan una transformación en la organización social de algunas de estas poblaciones, que ya no corresponden a familias campesinas, sino que se organizan estatalmente ligadas a la economía del Inca, la que se orienta principalmente a la producción de excedentes y tributos, integrándose durante el PT a una amplia red de circulación e intercambio. En definitiva, existe una modificación significativa de las comunidades locales en comparación con lo conocido para el PIT, caracterizado por un mayor aprovechamiento de los recursos disponibles, complejizando los asentamientos.

En el presente trabajo se abordan los resultados del análisis zooarqueológico realizado en 10 sitios asignados tanto al PIT como al PT con el fin de caracterizar a grandes rasgos estas ocupaciones en el Valle de Mauro. Más que una discusión sobre las tendencias en el registro de cada sitio, se realiza una síntesis con el objetivo de formular preguntas relacionadas al registro zooarqueológico y los alcances de estas evidencias para el PIT/PT.

\section{EL CASO DEL VALLE DE MAUROY SITIOS ESTUDIADOS}

Los sitios estudiados en este trabajo se ubican en el Valle de Mauro, comuna de Los Vilos, Provincia del Choapa, en la IV región de Coquimbo (ver Figura I). En este valle, los sitios se insertan en una red espacial que traspasa los límites costa-interior, conformando un sistema integrado de asentamientos Diaguitas que se redefinen durante el PT, manteniendo prácticas propias de las poblaciones Diaguitas preincaicas pero asociadas a una nueva lógica asociada al Tawantinsuyu (ver cronologías de sitio en Figura 2). Los análisis arqueofaunísticos realizados contaron con varias etapas, entre ellas cabe destacar la identificación anatómica, taxonómica y de rastros 
tafonómicos, entre otras. El detalle del análisis zooarqueológico, taxonómico y tafonómico de cada sitio se encuentra detallado en otros trabajos, así como las características generales de los contextos (véase Cartajenaet al. 2012, López et al. 2012, 2013, Santander y López 2012).

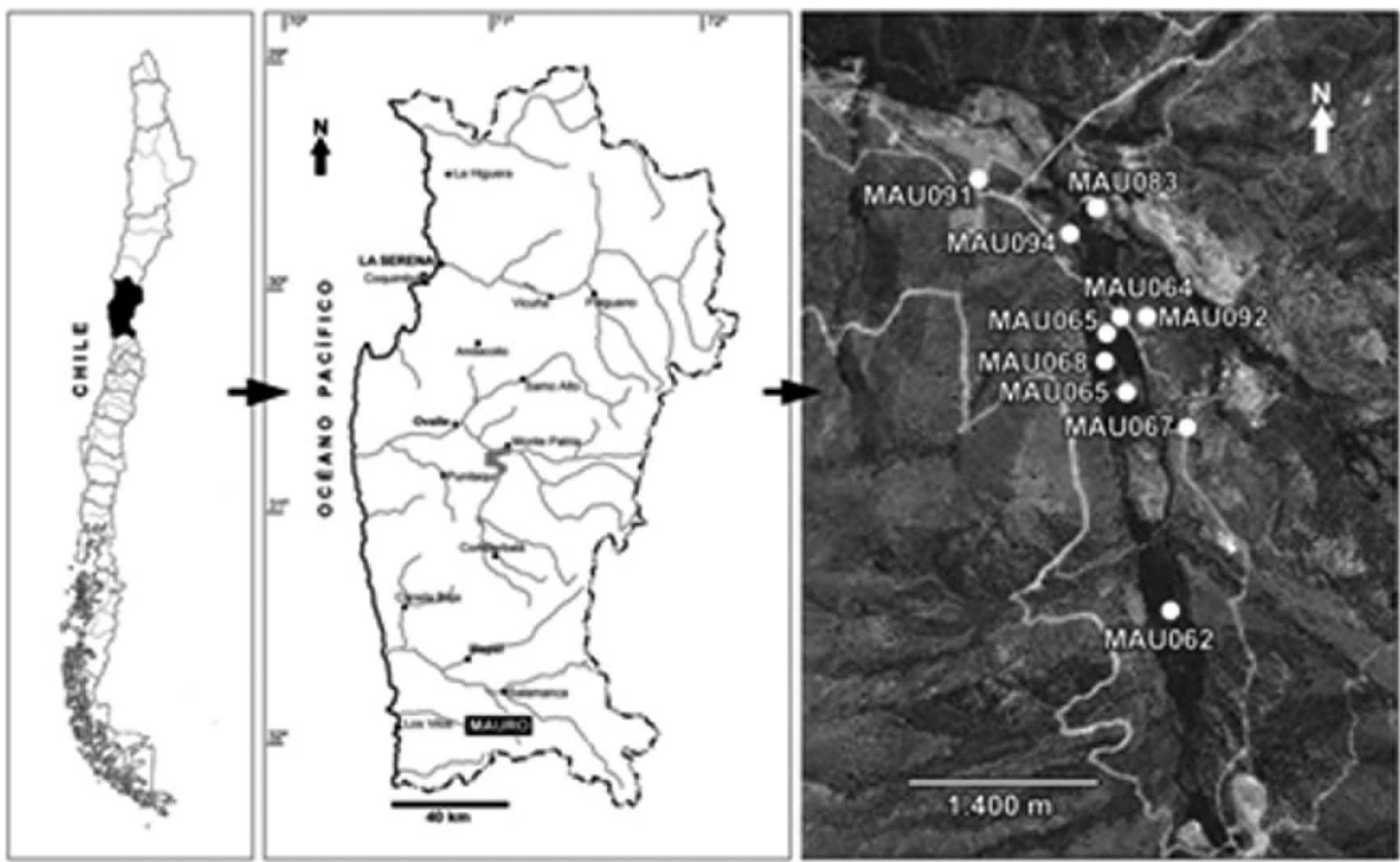

Figura 1: Ubicación del Valle del Mauro y los sitios mencionados en el texto (Imagen obtenida de Google Earth). Figure 1: Location of the Mauro Valley and the sites mentioned in the text (Image obtained of Google Earth).

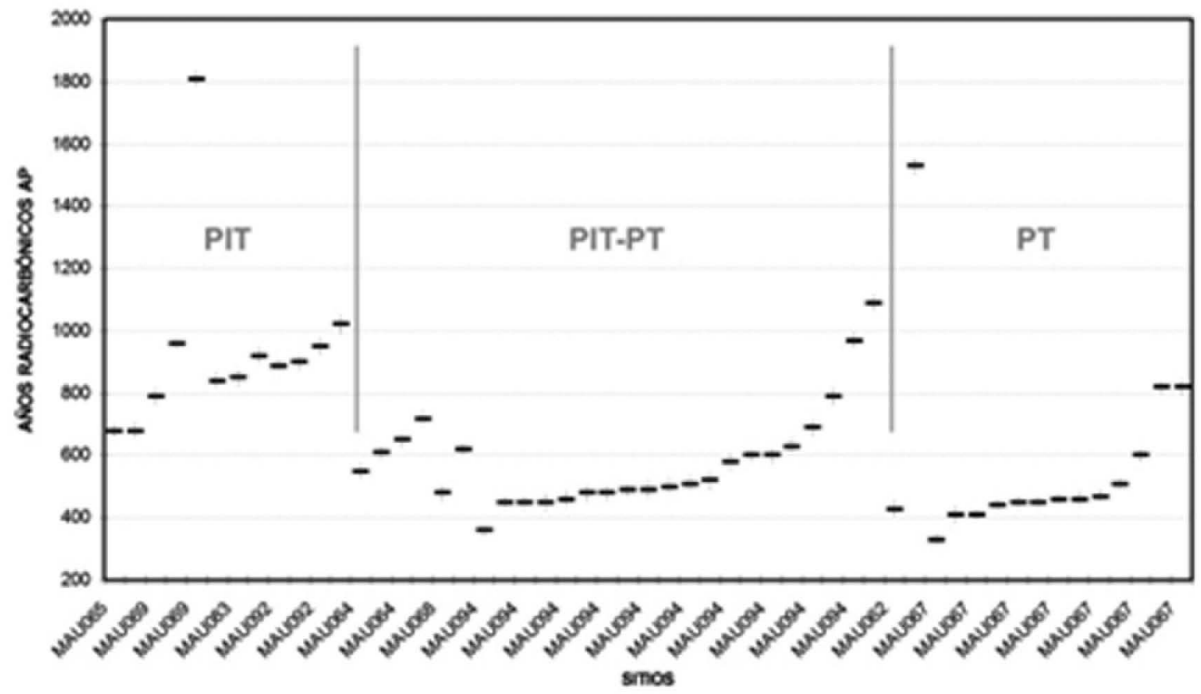

Figura 2: Fechas C14 (no calibradas) de los sitios estudiados.

Figure 2: ${ }^{14} \mathrm{C}$ dates (not calibrated) of the studied sites. 
En la Tabla I se resume la diversidad taxonómica de los sitios MAU065,MAU069,MAU083, MAU092 asignados al PIT; los sitios MAU064, MAU068, MAU094 del PIT/PT;y por último los sitios del PT, MAU062 y MAU067. Cabe destacar que la categoría PIT/PT corresponde a un criterio analítico el cual resume dos periodos que no fueron posibles de separar a partir del análisis zooarqueológico y tafonómico. Dentro de este resumen se observan las diferencias en la diversidad y abundancia de restos faunísticos destacando para el PIT sitios como MAU083 y MAU092 que si bien no son tan diversos, su registro es considerablemente mayor en comparación a sitios del mismo período en el valle, como MAU065 y MAU069. En el caso del PIT/PT el material se concentra, en términos de abundancia, en MAU094 en comparación con MAU064 y MAU068. Además, la diversidad es mayor en relación a los sitios asignados al PIT, diversificándose los restos de microfauna y mesofauna. Para el PT, esta diversidad se mantiene en parte, destacando el sitio MAU067 como el contexto con mayor número de evidencias. La abundancia y diversidad de restos faunísticos en los sitios mencionados se asocia además a un abundante registro de producción cerámica, artefactos líticos, y en el caso de MAU094, de artefactos metálicos, entre otros (Latorre 20II).

\begin{tabular}{|c|c|c|c|c|c|c|c|c|c|}
\hline \multirow[b]{2}{*}{ TAXA } & \multicolumn{4}{|c|}{ PIT } & \multicolumn{3}{|c|}{ PIT/PT } & \multicolumn{2}{|c|}{ PT } \\
\hline & $\begin{array}{c}\text { MAU } \\
065\end{array}$ & $\begin{array}{c}\text { MAU } \\
069\end{array}$ & $\begin{array}{c}\text { MAU } \\
083\end{array}$ & $\begin{array}{c}\text { MAU } \\
092\end{array}$ & $\begin{array}{c}\text { MAU } \\
064\end{array}$ & $\begin{array}{c}\text { MAU } \\
068\end{array}$ & $\begin{array}{c}\text { MAU } \\
094\end{array}$ & $\begin{array}{c}\text { MAU } \\
062\end{array}$ & $\begin{array}{c}\text { MAU } \\
067\end{array}$ \\
\hline Mammalia & 20 & 39 & 2863 & 964 & 0 & 47 & 26348 & 1198 & 17746 \\
\hline Camelidae & 2 & $I$ & 143 & 104 & 29 & 6 & 3823 & 25 & 1685 \\
\hline Abrocoma bennetti & 0 & 0 & 12 & 2 & 13 & 0 & 9 & I & 7 \\
\hline Ave & 0 & 0 & 5 & 0 & 1 & 0 & 0 & 0 & 0 \\
\hline Callopistes sp. & 0 & 0 & I & 0 & 0 & 0 & 26 & 0 & 40 \\
\hline Liolaemus sp. & 0 & 0 & 0 & 0 & 0 & 0 & 3 & 0 & I \\
\hline Canidae & 0 & 0 & I & 0 & 3 & 1 & 2 & 3 & 3 \\
\hline Lycalopex culpaeus & 0 & 0 & 0 & 0 & 0 & 0 & 0 & 0 & 2 \\
\hline Lycalopex griseus & 0 & 0 & 0 & 0 & 0 & 0 & 0 & 0 & 2 \\
\hline Carnivora & 0 & 0 & 0 & 0 & 0 & 0 & 12 & 0 & 0 \\
\hline Mustelidae & 0 & 0 & 0 & 0 & 0 & 0 & 0 & 7 & 0 \\
\hline Thylamys elegans & 0 & 0 & 0 & 0 & 0 & 0 & 0 & 0 & I \\
\hline Rodentia & 0 & 0 & 45 & I & 0 & 0 & 2441 & 4 & 0 \\
\hline Cricetidae & 0 & 0 & 0 & 6 & 0 & 0 & 0 & 0 & 0 \\
\hline Phyllotissp. & 0 & 0 & 0 & 0 & 0 & 0 & 8 & 0 & 2 \\
\hline Octodonsp. & 0 & 0 & 10 & 1 & 0 & 0 & 764 & 0 & 197 \\
\hline Spalacopus cyanus & 0 & 0 & 4 & 0 & 0 & 0 & 25 & 3 & 13 \\
\hline Lagidium viscacia & 0 & 0 & 3 & 2 & 3 & 0 & 4 & 0 & I \\
\hline Myocastor coypus & 0 & 0 & 0 & 0 & 0 & 0 & 0 & 0 & 3 \\
\hline Rhinellas pinulosa & 0 & 0 & 0 & 0 & 0 & 0 & 4 & 0 & I \\
\hline TOTAL & 22 & 40 & 3087 & 1080 & 49 & 54 & 33469 & | 24 | & 19704 \\
\hline
\end{tabular}

Tabla 1: NISP de especies nativas del valle presentes en los sitios estudiados (no se incluyen especies provenientes de la costa). Table 1: NISP from native species of the valley presents in the studied sites (species from the coast are not included).

Para abordar las tendencias en cada periodo, en la Tabla 2 se resumen algunas de las evidencias más importantes de cada sitio. Para el PIT, la presencia de camélidos domesticados no es del todo clara puesto que la diversidad de tamaño observada para el período (Cartajena et al. 20I2). Esta situación puede explicarse por procesos de crianza y no de domesticación propiamente tal. 
La Cultura Diaguita ha sido caracterizada como una sociedad campesina con un patrón de asentamiento disperso y explotación a baja escala de recursos (Troncoso 1999, 2001). Bajo este escenario, cabe preguntarse entonces si las evidencias de Valle de Mauro obedecen a este patrón de asentamiento descrito para otras zonas. En parte la respuesta es afirmativa, puesto que previo a la llegada del Tawantinsuyu y en comparación a las ocupaciones PIT la explotación de los recursos es a baja escala y menos diversificada. Se suma además la escasa producción de artefactos óseos para trabajos específicos, salvo aquellos utilizados para retoque de artefactos líticos y posiblemente los usados en textilería.

\begin{tabular}{|c|c|c|c|c|}
\hline Sitio & Asignación cultural & Tipo de sitio & Camélidos & Morfología artefactos* \\
\hline MAU065 & PIT & Doméstico & $\begin{array}{l}\text { Grupo grande } \\
\text { Indeterminado }\end{array}$ & - \\
\hline MAU069 & PIT & Doméstico & $\begin{array}{l}\text { Grupo grande } \\
\text { Indeterminado }\end{array}$ & - \\
\hline MAU083 & PIT & Doméstico & $\begin{array}{l}\text { Lama guanicoe } \\
\text { ¿Lama glama? }\end{array}$ & $\begin{array}{c}\text { Aguzado } \\
\text { Redondeado }\end{array}$ \\
\hline MAU092 & PIT & $\begin{array}{l}\text { Doméstico } \\
\text { Funerario }\end{array}$ & $\begin{array}{l}\text { Lama guanicoe } \\
\text { ¿Lama glama? }\end{array}$ & - \\
\hline MAU064 & $\mathrm{PIT} / \mathrm{PT}$ & Doméstico & Lama guanicoe & - \\
\hline MAU068 & $\mathrm{PIT} / \mathrm{PT}$ & Doméstico & $\begin{array}{l}\text { Grupo grande } \\
\text { Indeterminado }\end{array}$ & - \\
\hline MAU094 & $\mathrm{PIT} / \mathrm{PT}$ & $\begin{array}{l}\text { Doméstico } \\
\text { Funerario }\end{array}$ & $\begin{array}{l}\text { Lama guanicoe } \\
\text { Lama glama }\end{array}$ & $\begin{array}{c}\text { Aguzado } \\
\text { Aguzado romo } \\
\text { Redondeado } \\
\text { Adorno } \\
\text { Falanges perforadas } \\
\text { Astrágalo perforado }\end{array}$ \\
\hline MAU062 & PT & $\begin{array}{l}\text { Doméstico } \\
\text { Funerario }\end{array}$ & Lama guanicoe & - \\
\hline MAU067 & PT & $\begin{array}{l}\text { Doméstico } \\
\text { Funerario }\end{array}$ & $\begin{array}{l}\text { Lama guanicoe } \\
\text { Lama glama }\end{array}$ & $\begin{array}{c}\text { Aguzado } \\
\text { Aguzado romo } \\
\text { Redondeado } \\
\text { Biapuntado } \\
\text { Falanges perforadas }\end{array}$ \\
\hline
\end{tabular}

Tabla 2. Características de los sitios asignados al PIT, PIT/PT y PT.

* Sobre morfología de artefactos óseos en el Valle de Mauro véase Santander y López (2012)

Table 2: Characteristics of the sites assigned to PIT, PIT / PT and PT. ${ }^{\star}$ Regardingbone tools morphology in Mauro Valley, see Santander and López (2012)

Con la llegada del Inca al valle los cambios son evidentes, puesto que se diversifica la explotación de recursos locales, así como la diversidad de artefactos para trabajos de distinta índole destacando aquellos usados para trabajos textiles y otros de uso desconocido, como segundas falanges de camélidos con perforaciones en su diáfisis. Destaca además el uso de la llama, identificada con claridad en los sitios MAU094 y MAU067, que fue empleada como recurso económico, para articular zonas distantes y posiblemente como animales laneros.

\section{REFLEXIONES FINALES}

Durante el PT, la población Diaguita que habitaba estos sectores del NSA sufre una serie de cambios relacionados con la llegada del Inca a la región, implicando transformaciones económicas que se expresan en el registro arqueofaunístico de los sitios. En una primera instancia, en los sitios estudiados asignables a los períodos en cuestión es posible observar una mayor diversidad de taxa en comparación al PAT. No sólo existiría una dieta generalizada con recursos costeros, sino que además estos recursos van creciendo en cantidad y 
diversidad a través del tiempo, lo que probablemente se relaciona, durante el PIT/PT, con la necesidad de las poblaciones locales de diversificar sus recursos como dádivas al Inca. Así mismo, la posible aparición en el registro arqueológico de Lama glama a partir del PIT indica la realización de nuevas actividades a partir de nuevas necesidades. Este recurso, permitió construir una red de circulación de bienes, y por lo tanto, conectar estratégicamente distintos espacios, lo que se expresa por ejemplo en los emplazamientos de ciertos contextos, como el de MAU094. El registro faunístico permite, en tiempos tardíos, distinguir la funcionalidad que caracterizó a los sitios, como es el caso de los artefactos óseos que estarían indicando para el PT trabajo textil local, lo que implica el uso de fibras de animales tanto domésticos como silvestres (López et al. 20I2). De este escenario surge una interrogante, por cuanto los análisis morfométricos de los restos de camélidos para el PIT-PT indican una gran diversidad de tamaños que pueden interpretarse para el PIT como procesos iniciales de crianza, mientras que para el PIT/PT como la introducción de llamas y alpacas por parte del Inca (Cartajena et al. 2012) ¿Se asocia esta diversidad de tamaño a un aumento en la producción de textiles? Para el PT es un escenario plausible, considerando que los textiles son elementos relevantes para el Tawantinsuyu dentro de sus estrategias de reciprocidad y redistribución (Troncoso 2004). No obstante, es una pregunta que necesita ser abordada a futuro para sitios exclusivamente PIT. Si se observa además la distribución de taxa por período, para el PT aumenta la presencia de mustélidos, zorros, y roedores en general que puede explicarse no sólo por una mayor diversificación de la dieta sino de un uso más extensivo de pieles.
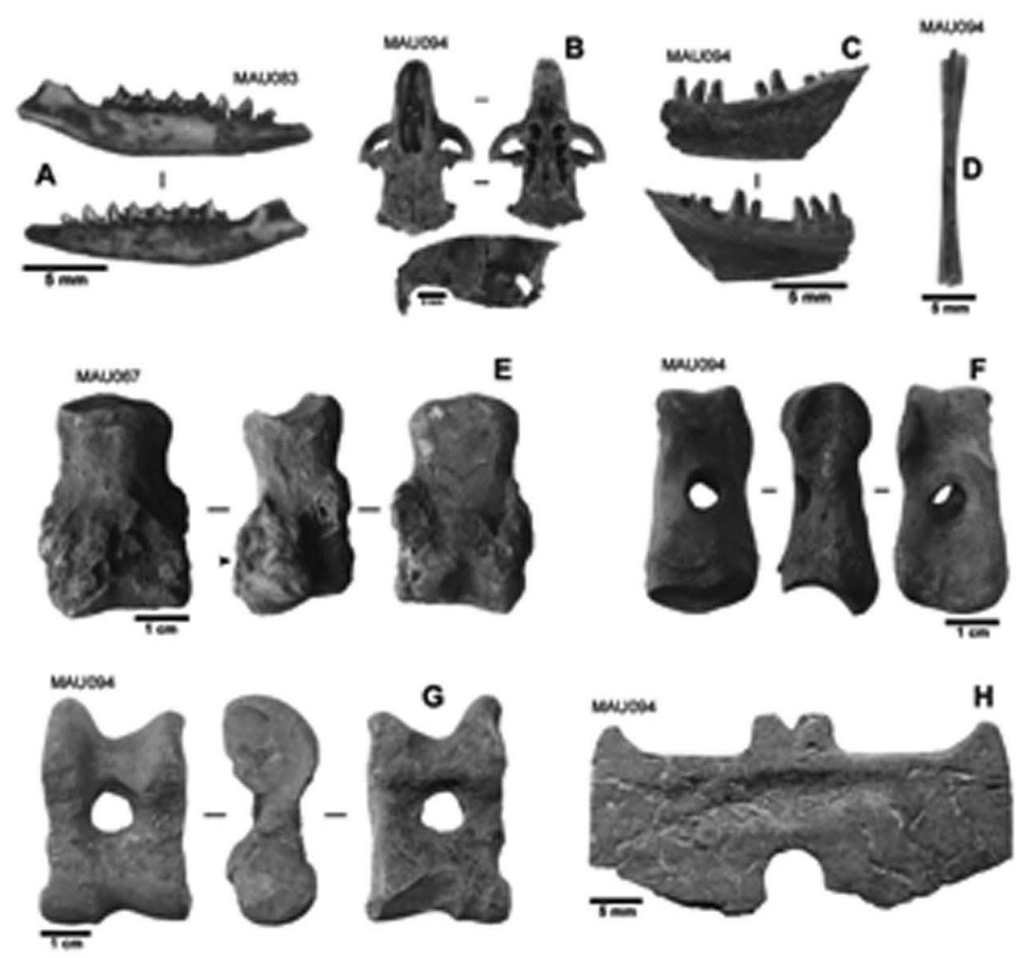

Figura 3: Restos faunísticos y artefactos recobrados de los sitios estudiados: a) Mandíbula de Thylamiselegans, b). Fragmento de cráneo de Octodonsp., c) Dentario de Callopistesmaculatus, d) Hueso de Rhinellaspinulosa, e) Falange de camélido con patología, f). Falange de camélido con perforación en su diáfisis, g) Astrágalo de camélido con perforación en su centro, h) Posible adorno corporal elaborado en hueso.

Figure 3: Faunal remains and bone artifacts recovered of the studied sites: a) Thylamiselegansmandible,b) Skull fragment of Octodon sp., c) Callopistesmaculatusroothwort, d) Rhinellaspinulosa bone, e) Camelid phalanx with pathology, $f$ ) Camelid phalan $x$ with perforation in his diaphysis, g) Camelidastragalus with perforation in his center, $h$ ). Possible corporal adornmentelaborated in bone. 
Dentro de las ocupaciones PT, un registro recurrente corresponde a segundas falanges de camélidos, con perforaciones en la diáfisis ubicadas en las superficies palmares y plantares (ver Figura 3f). La presencia de estos artefactos es exclusiva para este período y fueron registrados en basureros asociados a huesos de animales depositados postconsumo, en contextos similares a otros del semiárido, como Cesped-3 (Becker comunicación personal, Troncoso 2004). El conjunto artefactual óseo ve expandido en este período el repertorio de actividades desarrolladas, exhibiendo una elevada representación de artefactos dedicados al retoque por presión de material lítico ("compresores", que predominan en el conjunto), la perforación de cueros, las actividades en manufactura textil como se ha mencionado anteriormente $y$, aunque en menor medida, su utilización como colgantes.

Durante el PIT y PT se observa un cambio radical en el registro zooarqueológico del Valle de Mauro y en todo el NSA en comparación con periodos anteriores. La mayor diversidad de recursos tanto de la costa como interior se relaciona a cambios políticos y socioeconómicos relevantes, siendo uno de ellos la crianza e introducción de animales como la llama. Al respecto, en los sitios del PT aparece un morfotipo grande de llama fácilmente reconocible, mientras que para el PIT se observa una mayor variabilidad en el tamaño, dentro de un escenario más complejo. Este escenario es uno de los principales problemas de investigación a abordar en el futuro, requiriendo un cruce mayor de datos morfométricos, morfológicos, de dieta (isótopos estables) y genéticos, entre otros.

Agradecimientos: Nuestros agradecimientos a Daniel Pavlovic y Daniel Pascual por su ayuda durante el proceso de análisis. Esta investigación fue financiada por el Proyecto Arqueológico Mauro realizado por el Departamento de Antropología de la Universidad de Chile.

\section{BIBLIOGRAFÍA}

Becker, C.2004 “Animales que cuentan historias”. Chungará volumen especial: 359-364.

Belmar, C. y L. Quiroz. 2004 "Recursos vegetales y modos de explotación: ¿Qué nos dicen las plantas acerca de sus consumidores? Un estudio de sitios Diaguita en los valles de Illapel y Chalinga". Revista Werken (5): 53-60.

Cartajena, I, P. López, B. Santander y B. Rivera. 2012. Informe de los restos arqueofaunísticos del Valle del Mauro, IV Región, Chile. Manuscrito en poder de los autores.

González, P. 2004. "Patrones Decorativos y espacio: El arte visual diaguita y su distribución en la cuenca del río Illapel”. Chungará, 36 (volumen especial):767-78।.

Latorre, E.20I I. Informe Análisis de restos arqueológicos de metal. Sitios Valle El Mauro. Manuscrito en poder de la autora.

Latorre, E. y P. López. 20II. "Los metales en la cultura diaguita chilena (ca. 900-1536 DC): una aproximación metodológica e interpretativa”. Intersecciones en Antropología 155 167.

López, P., I. Cartajena, B. Santander, B. Rivera y C. Opazo. 20I2. "Explotación de camélidos de un sitio Intermedio Tardío (I.000-I.400 d.C.) y Tardío (I.400-I.536 d.C.) delValle de Mauro (IV Región, Chile)". Boletín de la Sociedad Chilena de Arqueología 4I-42:9|- 108.

López, P., I. Cartagena, B. Santander, D. Pavlovic y D. Pascual. 2013. "Camélidos domésticos en el Valle de Mauro (Norte Semiárido, Chile): múltiples análisis para un mismo problema”. Intersecciones en Antropología. En prensa.

Santander, B. y P. López. 2012."Análisis de Microhuellas de Uso Mediante Microscopio Electrónico de Barrido (MEB) de Artefactos Óseos de un Sitio Arcaico Tardío del Valle de Mauro (Región de Coquimbo, Chile): Aportes para una Reconstrucción Contextual”. Revista Chilena de Antropología 26: I29-I50.

Troncoso, A. 1999. "La Cultura Diaguita en el valle de Illapel: Una perspectiva exploratoria”. Chungará 30 (2): I25-I42.

Troncoso, A. 2001. "La cultura Diaguita en el 200I, problemas y perspectivas desde el Choapa”. Actas del IV Congreso Chileno de Antropología, tomo II, PP. I35I-1356. Colegio de Antropólogos de Chile, Santiago.

Troncoso, A. 2004. "Relaciones socio-culturales de producción, formas de pensamiento y ser en el mundo: un acercamiento a los períodos Intermedio Tardío y Tardío en la cuenca del río Choapa". Revista Werken 5: 6I-68. 